\title{
XXXII.
}

(Aus dem Institut für Krebsforschung in Heidelberg. Direktor: Wirklicher Geheimer Rat Prof. Dr. V. Czerny, Exz.)

\section{Ueber den Einfluss von Thermalinjektionen auf das Mäusecarcinom.}

\author{
Von \\ Privatdozent Dr. R. Werner, I. Assistenzarzt am Samariterhause.
}

Die Versuche von Clowes und Bäslack, sowie von Michaelis haben ergeben, dass man Tumorstückchen, die von transplantablen Mäusecarcinomen entnommen sind, durch längeres Erwärmen auf eine bestimmte Temperatur, welche im Einzelfalle zwischen $37^{\circ}$ und $45^{\circ}$ schwankt, für die Uebertragung geeigneter machen kann, indem dann die überimpften Zellmassen nicht nur leichter, d. h. in einem grösseren Prozentsatze angehen, sondern auch dauernd rascher wachsen, also in ibrer Malignität merklich gesteigert erscheinen. Eine Erhitzung über den für die betreffende Geschwulstart optimalen Grad hinaus führte zur Herabsetzung der Transplantationsfähigkeit, wie der Wachstumsgeschwindigkeit. Es war nun zu untersuchen, ob im Mäusekörper eine ähnliche Beeinflussung des Krebsgewebes durch Erhitzung möglich ist, wie in vitro. Als Thermophor wurde sterile, physiologische Kochsalzlösung gewählt, weil dadurch einerseits Komplikationen mit chemischen oder bakteriellen Einflüssen vermieden, andererseits infolge der hohen Wärmekapazität des Wassers beträchtliche Energiemengen appliziert werden konnten. Die Näusetumoren wurden mit relativ grossen Quantitäten der auf einen bestimmten Temperaturgrad erhitzten Kochsalzlösung infiltriert, wobei entweder der Uebertritt der Flüssigkeit in das normale Körpergewebe soviel als möglich eingeschränkt, oder umgekehrt, die Geschwulst absichtlich auch rings im Gesunden umspritzt wurde. Die Einführung der Flüssigkeit geschah mit Hilfe einer zur Vermeidung von Wärmeverlusten vorgewärmten sterilisierten Metallspritze meist direkt von der enthaarten und desinfizierten Wölbung der Tumoren aus, nur einige 
Nale wurde zum Vergleich durch die benachbarte Haut in das normale Gewebe eingestochen. Die Geschwülste entstammten einem von Bashford (London) dem Krebsinstitute äberlassenen und auf Wiener Mäusen erfolgreich weitergezüchteten, rasch wachsenden Tumor von alveolärer Struktar ${ }^{1}$ ). Ihr Umfang variierte in beträchtlichem Masse; die kleinsten waren bohnen-, die grössten walnussgross. Sie waren alle geschlossen und von intakter Haut bedeckt, derb elastisch, ohne palpatorisch nachweisbare Erweichungen.

Es wurden 1,3 oder $6 \mathrm{ccm}$ für jede Injektion verwendet und Temperaturen von $50^{\circ}, 55^{\circ}, 60^{\circ}, 65^{\circ}, 70^{\circ}$ und $80^{\circ}$ Celsius auf ihre Wirkung: geprüft. Bei einer Einspritzung von $1 \mathrm{ccm}$ blieb die Flüssigkeitsmenge bis auf einen kleinen Tropfen, der aus dem Stichkanale quoll, im Tumorgewebe; die Injektionen von 3 oder $6 \mathrm{ccm}$ führien, wenn sie von einem Stichkanale aus gemacht wurden, nicht nur zu einer Infiltration der Geschwulst, sondern auch zu einer solchen der Umgebung. Wurde mehrere Male in das Carcinom gestochen, so strömte ein Teil der Flüssigkeit durch die ersten Stichkanäle wieder aus, der Tumor wurde dann gewissermassen mit heisser Kochsalzlösung durchgespült, nicht nur mit ihr infiltriert. Jedes Experiment wurde $3-4 \mathrm{mal}$ wiederholt. Dabei genügte der Widerstand der Tumorkapsel, um den Austritt in die Umgebung zu verhindern.

Die Ergebnisse waren bei denselben Versuchsanordnungen immer die gleichen. Diese Koustanz dürfte im wesentlichen dem Umstande zu danken sein, dass die Testobjekte alle einem einheitlichen Tumorstamme und einer einzigen Mäuserasse angehörten. Die Frage, inwieweit eine diesbezügliche Variation die Resultate beeinflussen würde, kann allerdings auf Grund des vorliegenden Materiales nicht beantwortet werden.

Es sollen nun die Beobachtungen in aller Kürze, nach Temperaturgrad and Flüssigkeitsmenge geordnet, mitgeteilt werden.

\section{$50^{\circ} \mathrm{Kochsalzlösung:} 1 \mathrm{ccm}$.}

Wirkung: Die Geschwalst nimmt in den nächsten $3-4$ Tagen rascher zu als dies vor der Injektion der Fall war. Der Unterschied ist jedoch nicht sehr gross. Keine Veränderung der Umgebung.

$50^{\circ}$ Kochsalzlösung : $3 \mathrm{ccm}$.

Durchspülung der Geschwulst ergibt denselben Effekt, wie bei Infiltration mit $1 \mathrm{ccm}$, Injektion ohne Abfiessen der Flüssigkeit vom 2. Tage an eine viel raschere Volumzunahme des Tumors, die 2-3 Tage anbält. Geschwulst dann etwa auf das $\mathbf{1} 1 / 2$ fache vergrössert. Umgebung

1) Adenoma malignum mamnae. 
R. Werner, Einfluss von Thermalinjektionen auf das Mäusecarcinom.

unverändert. Später eine weniger auffällige Beschleunigung des Wachstums.

500 Kochsalzlösuug: $6 \mathrm{cem}$.

Bei Durchspülung der Geschwulst eine ähnliche Wirknng, wie nach Injektion der halben Menge. Nach Injektion von $6 \mathrm{cem}$ ist die Wucherung viel stärker, in 3-4 Tagen den Umfang des Tumors auf das Doppelte steigernd. Keine Veränderung der Umgebung.

\section{$55^{0}$ Kochsalzlösung : 1 cem.}

Zunächst eher eine kleine Schrumpfung der Geschwulst, dann aber vom 3. Tage ab eine beträchtliche Volumzunabme, etwa bis zur $11 / 2$ fachen Grösse. Umgebung unverletzt.

\section{$55^{\circ}$ Kochsalzlösung: $3 \mathrm{ccm}$.}

Nach Infiltration zuerst eine deutliche Schrumpfung, dabei auch Erweichung des Tumors, ohne deutliche Fluktuation. Vom 3. Tage an rapide Wucherung. Umgebung mässig entzündet. Durchspülung hat nur eine geringe primäre Verkleinerung zur Folge, aber fast ebenso rasche und beträchtliche Volumzunahme. Umgebung dabei unverändert.

\section{$55^{0}$ Kochsalzlösung : $6 \mathrm{ccm}$.}

Primäre Schrumpfung und sekundäres Nachwachsen noch mehr ausseprägt, als bei den halben Mengen, ebenso die Entzündung der Umgebung nach der Infiltration. Kleine Nekrosen der Umgebung des Stichkanales.

Bei Anwendung von $60^{\circ}$ und $65^{\circ}$ Kochsalzlösungen sind die Wirkungen so ziemlich identisch.

1 cem.

Nach der Infiltration kommt es rasch zu einer Schrumpfung und Erweichung des Tumors, dagegen ist das Nachwachsen entschieden verzögert (erst vom 5. bis 6. Tage an) und bei kleineren Tumoren nur in mässigem Grade zu konstatieren, bei grossen wächst die Peripherie schon vom 3. Tage an rapid weiter. Umgebung nicht verändert.

\section{$3 \mathrm{ccm}$.}

Nach Infiltration Erweichung des Tumors bis zur Fluktnation. Volumverminderung in 2-3 Tagen bis auf die Hälfte, ja auf $1 / 3$. Geringfügige zentrale Nekrose. Das Wachstum des Tumors bleibt einige Zeit hindurch deutlich abgeschwächt, erst nach etwa 8-10 Tagen allmähliches Nachvachsen. Umgebung stark entzündet, zeigt jedoch nur vereinzelte, kleine 
Epidermisuekrosen. Bei Durchspülung dieselben Erscheinungen weniger ausgeprägt, Neigung zum Nachwachsen rom 5. bis 6. Tage an. Umgebnng nur schwach entzündet.

\section{כ $\mathrm{cem}$.}

Bei Infiltration rasche Rückbildung der Geschwulst unter vorübergehender, bis zur Fluktuation fortschreitender Erweichung mit kleiner zentraler Nekrose. Kein deutliches Nachwachsen. Umgebung heftig entzündet, aber nur sporadisch in den obersten Hautschichten nekrotisiert. Bei Durchspülung des Tumors die gleiche Kolliquation und Schrumpfung, aber fast ohne Nekrosen und mit geringer Entzündung der Umgebung.

\section{Kochsalzlösung.}

Die Wirkung unterscheidet sich von der bei $65^{\circ}$ nur durch etwas stärkere Nekrotisierung an den Finstichstellen und die beträchtlichere Reaktion der Umgebung. In einem Falle kommt es nach einmaliger Durchspülung mit $6 \mathrm{~cm}$ zu einem kompletten Schwunde der Geschwulst, an deren Stelle sich nach 14 Tagen eine rasch zuheilende, schön granulierende Wunde befindet.

\section{$80^{0} \mathrm{Kochsalzlösung.}$}

Bei $1 \mathrm{ccm}$ entsteht eine grosse, zentrale Nekrose, in deren Nachbarschaft der Tumor erweicht, doch nur wenig schrumpft. Bei $3 \mathrm{ccm}$ hat die Infiltration, wie die Durchspülung, eine Totalnekrose der Geschwulst und ihrer Umgebung zur Folge, was zur Abstossung ohne Schrumpfung führt. $6 \mathrm{ccm}$ bewirken dieselben Erscheinungen in noch höherem Grade, töten aber die Mäuse.

2 Tiere starben nach Injektion von $60^{\circ}$ und $70^{\circ}$ Kochsalzlösung an Nebenverletzungen. In beiden Fällen war die Flüssigkeit bis in die Bauchhöhle vorgedrungen und hatte das Peritoneum verbrüht.

Es ergibt sich somit die auffallende Tatsache, dass bei Injektion ron $50-55^{\circ}$ Kochsalzlösung die Tumoren zu rascherem Wachstum gereizt, bei Anwendung von $60-70^{\circ}$ erweicht, verflüssigt und zur Schrumpfung gebracht, bei $80^{\circ}$ aber nekrotisiert werden. Die normalen Gewebe der Umgebung leiden bedeutend weniger als die Geschwülste selbst, auch dann, wenn ein nicht unbeträchtlicher Teil der Flüssigkeit sie mitimbibiert. Selbst wenn man berïcksichtigt, dass letztere erst in die Tumormasse gelangte und dort etwas abgekühlt wurde, ehe der Austritt in die Nachbarschaft erfolgte, kanu man sich doch des Eindruckes nicht erwehren, dass die Geschwulstelemente gegen die thermische Wirkung sensibler sind, als die physiologischen Zellen des Körpers. Durch einige Kontrollversuche liess sich dies besser dartun: Wurden $3 \mathrm{ccm} 60^{\circ}$, oder $70^{\circ}$ oder $80^{\circ} \mathrm{Kochsalz}$ - 
lüsung dicht am Raude des Tumors in die Umgebung eingespritzt, so entstand eine ziemlich beträchtliche Entzündung in der Haut der Nachbarschaft, die aber nur stellenweise kleine Epidermisinseln verlor, während der Tumor von der Peripherie her rasch erweichte und einschmolz.

Für die mikroskopische Untersuchung fertigte mir Herr Dr. Zeller eine Reihe von mit Hämatoxylineosin gefärbten Schnitten an. Ich möchte ihm an dieser Stelle meinen verbindlichsten Dank aussprechen. Es zeigte sich, dass die makroskopisch zu differenzierenden Reaktionsarten, die Schrumpfung mit oder ohne Verflüssigung, sowie die nicht resorbierbare Nekrotisierung im histologischen Bilde ebenfalls deutlich zu trennen sind. Den Nekrosen entsprechen homogene oder grobstreifige Plasmamassen, die ron Kemtrümmern, spärlichen Leukozyten, an manchen Punkten auch von Erytbrozyten durchsetzt sind. Zellgrenzen sind nur an einzelnen Stellen schattenhaft erhalten, ebenso ist die Struktur der Gefässe nur angedeutet. In den erweichten schrumpfenden Tumorpartien fehlen die Zeichen der Gerinnung, die in den nekrotischen vorherrschen, fast vollständig. Die Zellen haben ein trübes, von zahlreichen Vakuolen durchsetztes Protoplasma, vielfach ideformierte oder in Zerfall begriffene Kerne; abgesehen von lokalen Hämorrhagien sind die Gefässe erhalten. Wir haben das Bild einer Gewebseinschmelzung unter intrazellulärer Höhlenbildung vor uns. Dabei sind die Gewebsspalten alle erweitert und von einer durchsichtigen, nur spärlich Leukozyten enthaltenden Flüssigkeit erfüllt, so dass die fluktuierenden Stellen der Geschwülste cystischen Degenerationen ähneln. Diese ödematöse Durchtränkung ist als eine Folge der entzündlichen Reaktion, nicht als der Rest der eingespritzten Flüssigkeit aufzufassen, da diese stets nach wenigen Stunden resorbiert war, während die Erweichung erst später einsetzte. Auch dort, wo rapide Wucherung angeregt wurde, ist das Protoplasma vieler Zellen nicht intakt, sondern leicht getrübt und vakuolisiert, ferner ist Gewebsödem und Hyperämie mit vereinzelten Hämorrhagien vorhanden, aber die Veränderungen sind viel geringgradiger als an den Punkten der Rückbildung des Tumors.

Es unterliegt keinem Zweife], dass wir hier ein klassisches Beispiel für das alte Gesetz vor uns haben, nach dem schwache Traumen das Wachstum der Zellen anregen, stärkere aber deren Untergang herbeifülhren.

Mit den Versuchen von Fuerst, der bei der Epidermis von Kaninchenobren die kritische Temperatur für Wucherung and Nekrose beträchtlich tiefer und näber aneinanderliegend fand, lassen sich diese Experimente nicht direkt vergleichen. Dort wurden die Zellen in grosser Flächenausdehnung mit einer ror Abkühlung sorgfältig geschützten Flüssigkeit in Berührung gebracht, hier aber musste nach dem Eintritte in das Gewebe sofort eine Temperaturerniedrigung erfolgen. Daher kam es nur in der 
Zone des ersten Temperaturausgleiches - um die Stichkanäle berum von $55^{\circ}$ aufwärts zu Nekresen, und zwar je höher der Wärmegrad war, bei um so kleineren Dosen. Ferner waren bei den Fuerstschen Versuchen die Zellen minutenlang demselben Temperaturgrad ausgesetzt, bei meinen Experimenteu infolge der Abkühlung im Körper nur sekundenlang.

Praktisch sind die Ergebnisse höchstens insofern zu verwerten, als man in den Thermalinjektionen ein Nittel hat, um die für die Kankrologie so wichtigen transplantablen Tumoren zu rascherem Wachstume anzuregen, was an unserem Institute auch Herrn Dr. Deetjeu wiederholt mit dem gewünschten Erfolge gelang. Dagegen ist zunächst ein Vorteil für die Therapie der Krebse noch nicht abzusehen, denn eiumal ist es nicht erwiesen, dass alle Geschwülste ebenso empfindlich sind, wie die von mir benutzten Mäusetumoren, dann lassen sich die meisten sicher nicht so gut infiltrieren, ferner besteht immer die Gefahr durch Reizung der nicht genügend getroffenen Tumorpartien erheblich zu schaden, und endlich wärden die Injektionen Narkosen erfordern, die in verhältnismässig kurzen Zwischeuräumen wiederholt werden müssten. Das Verfahren könnte nur dort in Frage kommen, wo man sicher in kurzer Zeit, womöglich mit einem Schlage, das kranke Gewebe vernichten könnte, d. h. bei zirkumskripten, kleinen Tumoren, und dort dürfte wohl die chirurgische Behandlung zuverlässiger sein. 